\title{
COMMENT
}

\section{Nitric oxide and preterm resuscitation: some words of caution}

\author{
Maximo Vento $\mathbb{i D}^{1,2}$ and Ángel Sánchez-Illana ${ }^{2}$ \\ Pediatric Research (2020) 87:438-440; https://doi.org/10.1038/s41390-019-0649-4
}

This article has been corrected since Advance Online Publication and a correction is also printed in this issue

Stabilization of preterm babies in the delivery room frequently requires positive pressure ventilation and oxygen supplementation to overcome lung immaturity and respiratory insufficiency. The initial inspired fraction of oxygen $\left(\mathrm{FiO}_{2}\right)$ has to be titrated according to oxygen saturation pulse oximetry $\left(\mathrm{SpO}_{2}\right)$ readings to keep oxygenation within a "safety" range that avoids hyperoxemia or hypoxemia. Despite numerous studies, both the optimal initial $\mathrm{FiO}_{2}\left(\mathrm{iFiO}_{2}\right)$ and titration procedure have not been yet clearly established. ${ }^{1}$ During postnatal stabilization, the use of $100 \%$ oxygen in term babies has been associated with increased oxidative stress and mortality, ${ }^{2}$ and in preterm infants with increased oxidative stress and higher incidence of bronchopulmonary dysplasia, need for oxygen, and length of hospital stay. ${ }^{3,4}$ International guidelines recommend $0.21 \mathrm{iFiO}_{2}$ for term and 0.3 for preterm infants. ${ }^{5}$ Besides, there are serious concerns that the use of lower $\mathrm{iFiO}_{2}$ would cause hypoxemia and/or bradycardia in the first minutes after birth and thus increase the risk of death and/or intraventricular hemorrhage (IVH). Oei et al. ${ }^{6}$ reviewed $\mathrm{SpO}_{2}$ and heart rate data from 768 infants $<32$ weeks gestation randomized to an initial $\mathrm{FiO}_{2}$ of 0.3 vs. 0.6 targeted to specific predefined $\mathrm{SpO}_{2}$ before $10 \mathrm{~min}$ of age and concluded that not reaching a $\mathrm{SpO}_{2}$ of $80 \%$ at 5 min was associated with death or IVH attributable either to infant's prenatal illness or insufficient oxygen upon resuscitation. Under these circumstances, neonatologists hesitate between providing lower $\mathrm{iFiO}_{2}$ with increased risk of death or IVH or higher $\mathrm{iFiO}_{2}$ causing oxidative stress. Solving this conundrum would require achieving an adequate oxygenation in the first minutes after birth with the least supplemental oxygen to avoid the risks inherent to hyperoxemia or hypoxemia.

In the current issue of Pediatric Research, Sekar et al. ${ }^{7}$ report on a pilot, double-blind, randomized, placebo-controlled trial enrolling 28 (14 per group) preterm infants with gestational age ranging from $250 / 7$ to $316 / 7$ that required positive pressure ventilation and oxygen in the delivery room. For the first time in neonatology, inhaled nitric oxide (iNO) in combination with oxygen have been used for postnatal stabilization. Ventilation was initiated either with $\mathrm{iFiO}_{2} 0.3+\mathrm{iNO}$ at 20 p.p.m. (intervention group) or $\mathrm{FiO}_{2} 0.3$ + nitrogen (placebo group). $\mathrm{SpO}_{2}$ was titrated according to current guidelines, ${ }^{5}$ while iNO was weaned at the end of the resuscitation period. The most compelling finding was the lower $\mathrm{FiO}_{2} \mathrm{~S}$ needed by the iNO group along stabilization that turned out in a significantly reduced rate of exposure to high $\mathrm{FiO}_{2}(>0.6)$. No differences in Apgar score at $5 \mathrm{~min}$, pre- and postductal saturations, $\mathrm{SpO}_{2}$, or need for intubation in the early neonatal period were recorded. No information regarding delayed cord clamping is provided. There was a tendency towards higher hazard ratio in the placebo group that could be interpreted as a compensatory reaction to increase the provision of oxygen to tissue. No differences in mortality, length of stay, days on mechanical ventilation, or neonatal conditions, such as retinopathy of prematurity, bronchopulmonary dysplasia, $\mathrm{IVH}$, or others were reported. ${ }^{7}$ Sekar et al. ${ }^{7}$ concluded that the use of iNO is feasible without any side effects and reduces the exposure to high levels of oxygen. The study has obvious limitations. It was stopped before reaching the calculated sample size due to difficulties in recruitment not reaching the statistical power required. In addition, no biomarkers of nitrosative stress were determined and no long-term follow-up results have been included in the publication. Thus, this novel approach to preterm stabilization stirs up several questions. Does the use of iNO entail risks derived from the generation of reactive nitrogen species (RNS)? Oxygen supplementation leads to the formation of reactive oxygen species (ROS). ${ }^{1}$ Nitric oxide ('NO) is a relatively stable and highly diffusible free radical. NO-mediated pathogenicity depends on the formation of highly reactive and toxic byproducts such as peroxynitrite anion $\left(\mathrm{ONOO}^{-}\right)$and nitrogen dioxide $\left({ }^{\circ} \mathrm{NO}_{2}\right)$. The formation of RNS requires the interaction of 'NO with oxidants such as superoxide radicals, hydrogen peroxide, and transition metals such as iron or copper. ${ }^{8}$ Of note, the rate constant of the reaction of anion superoxide $\left(\mathrm{O}_{2}{ }^{-}\right)$with ${ }^{\circ} \mathrm{NO}$ is 10 -fold higher than that of the SOD-catalyzed dismutation, and therefore nitric oxide kinetically outcompetes SOD for anion superoxide. ${ }^{8}$ Figure 1 depicts the equilibrium of peroxynitrite with peroxynitrous acid $(\mathrm{ONOOH})$ and the direct and indirect reactions that cause protein tyrosine nitration, protein oxidation, and DNA oxidation and nitration. ${ }^{8}$ However, iNO has been successfully employed for the treatment of respiratory diseases in term and preterm infants using a similar dose than that employed in the present study (20 p.p.m.) without toxic or unwanted side effects. ${ }^{9}$ Moreover, Ballard et al. ${ }^{10}$ demonstrated that iNO supplied at 20 p.p.m. compared to placebo did not increase plasma protein 3-nitrotyrosine and protein carbonylation, both markers of oxidative and nitrosative stress, respectively. However, patients studied by Ballard et al. ${ }^{10}$ underwent a completely different pathophysiological situation with respiratory distress still requiring ventilatory support and oxygen between days 7 and 21 after birth. Herewith, to minimize the amount of RNS formation and especially ${ }^{*} \mathrm{NO}_{2}$, the lowest necessary concentration of iNO and oxygen should be employed. In addition, $\mathrm{iNO}_{2}$ should be supplied into the ventilatory circuit as close as possible to the patient to reduce the time allowing for ${ }^{\circ} \mathrm{NO}_{2}$ formation. ${ }^{9}$

Nowadays, the most trustable nitrosative stress biomarkers are represented by nitrotyrosine, and proteins exclusively nitrated endogenously, such as nitroalbumin, which has been shown to correlate with the severity of neonatal encephalopathy and perinatal asphyxia. ${ }^{11}$ It would be very informative to confirm the presence of nitrosative and/or oxidative stress with reliable biomarkers such a nitroalbumin and/or 3-nitrotyrosine

${ }^{1}$ Division of Neonatology, University and Polytechnic Hospital La Fe, Valencia, Spain and ${ }^{2}$ Health Research Institute La Fe, Valencia, Spain

Correspondence: Maximo Vento (maximo.vento@uv.es)

Received: 23 August 2019 Revised: 12 October 2019 Accepted: 15 October 2019

Published online: 30 October 2019 


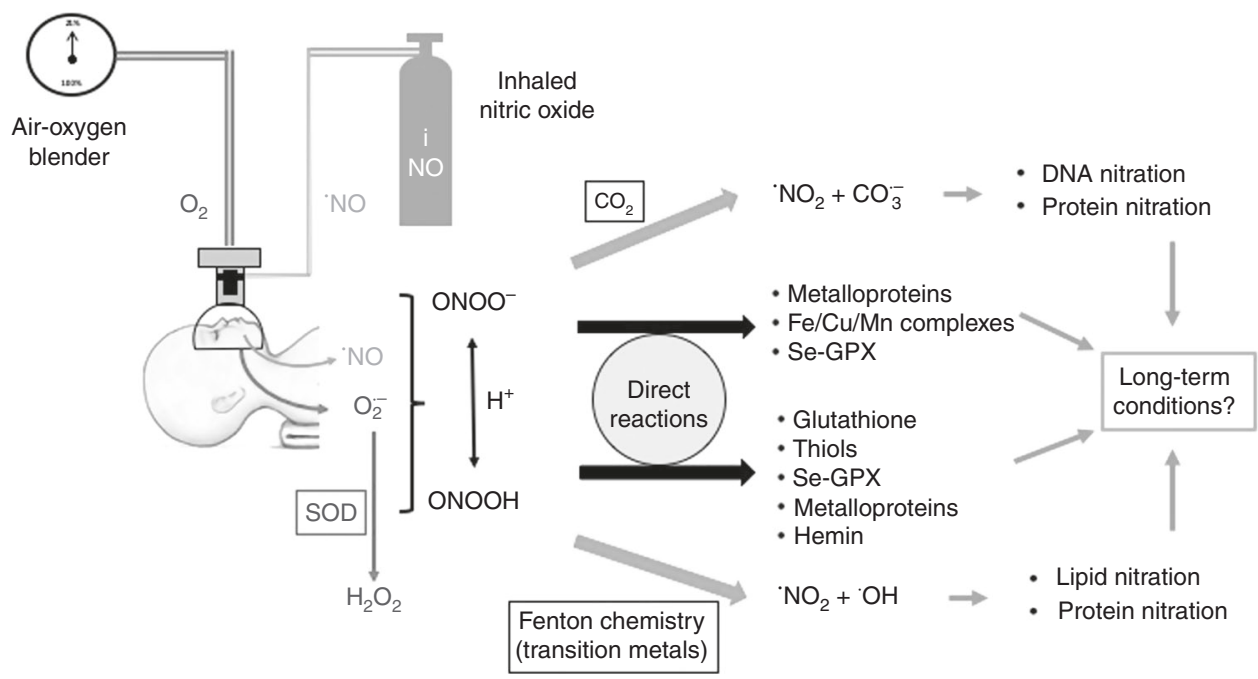

Fig. 1 Nitric oxide ( $\mathrm{NO})$ reacts with anion superoxide $\left(\mathrm{O}_{2}{ }^{--}\right)$forming peroxynitrite $\left(\mathrm{ONOO}^{-}\right)$outcompeting superoxide dismutation by superoxide dismutases (SOD). Peroxynitrite in the presence of protons achieves an equilibrium with peroxynitrous acid (ONOOH). Both these compounds directly react with a series of antioxidants, enzymatic complexes with transition metal core, metalloproteins, hemin, and so on. In addition, in the presence of carbon dioxide $\left(\mathrm{CO}_{2}\right)$, peroxynitrite forms highly reactive nitrogen dioxide and carbonate radicals, which directly react with DNA and proteins. In addition, peroxynitrous acid in the presence of hydroxyl radicals ("OH) generated through Fenton chemistry leads to the formation of nitrogen dioxide, which directly attacks lipids and proteins (based on ref. ${ }^{8}$ )

and F2-isoprostanes and isofurans, respectively, using validated mass spectrometry techniques to establish if there is a correlation between these markers and long-term outcomes.

Is methemoglobinemia influenced by delayed cord clamping? Under physiological conditions hemoglobin is oxidized to methemoglobin (metHb) reaching standard concentrations $<1.5 \%$ of total hemoglobin. Deleterious effects of rising metHb concentrations are associated with concomitant decreased oxygen carrying capacity in comparison to hemoglobin. ${ }^{12}$ No information regarding metHb is given in the study by Sekar et al. ${ }^{7}$ However, previous studies have shown that exposure to iNO at 20 p.p.m. or less are well tolerated in subjects with an adequate metHb reductase activity. ${ }^{9}$ It could be speculated that delaying cord clamping in the delivery room would increase the amount of circulating hemoglobin and reduce toxic concentrations of metHb. Another question is if iNO or just $\mathrm{FiO}_{2}$ be titrated during resuscitation to avoid hyperoxemia. No clearly defined standards for titrating oxygen during postnatal stabilization have been validated. ${ }^{1}$ For practical purposes, it seems adequate to adjust $\mathrm{FiO}_{2}$ while keeping iNO at a fixed concentration around 20 p.p.m. Rapid changes in iNO concentration should be avoided because they may cause a rebound pulmonary vasoconstriction leading to hypoxemia. ${ }^{9}$

To end up, follow-up studies are mandatory to assess if the use of iNO in the delivery room can have long-term consequences. In a recent meta-analysis, Barrington et al. ${ }^{13}$ did not find neurodevelopmental or sensorial impairment at 2 years in preterm infants treated with iNO in the neonatal period. However, Dixon et al. ${ }^{14}$ studied the association between neonatal intensive care unit therapies and childhood cancer. As a result of the multivariate logistic regression analysis, the only therapy that was independently associated with increased risk of cancer was iNO (adjusted odds ratio $8.6,4.3-17.4) .{ }^{14}$ This association should, however, be cautiously interpreted. To end up, it should be underscored that the complexity of the technical and training requirements for using iNO in the delivery room should be restricted to research purposes until further studies are put forward.

In summary, the study by Sekar et al. ${ }^{7}$ represents a fascinating conceptual breakthrough in preterm newborn resuscitation that aims to reduce the oxygen load provided during postnatal stabilization and avoid damage, especially caused by hypoxia. The use of iNO at 20 p.p.m. has been safely employed in the treatment of preterm infants. ${ }^{9}$ However, fetal-to-neonatal transition represents a special physiologic situation during which a series of complex respiratory, hemodynamic, and metabolic changes occur very rapidly. ${ }^{1}$ The management of oxygen and iNO in the delivery room should be closely monitored to avoid fluctuations in lung vascular tone. Finally, research on the use of iNO for resuscitation of preterm infants should include neurodevelopmental and clinical follow-up, and the inclusion of validated biomarkers of oxidative and nitrosative stress during the first hours after birth.

\section{ACKNOWLEDGEMENTS}

M.V. and A.S.-I. acknowledge PI17/0131 and FI16/00380 grants, respectively, from the Instituto de Investigación en Salud Carlos III (Ministry of Science, Innovation, and Universities, Kingdom of Spain).

\section{AUTHOR CONTRIBUTIONS}

M.V. and Á.S.-I. drafted the manuscript and approved its final version.

\section{ADDITIONAL INFORMATION}

Competing interests: The authors declare no competing interests.

Publisher's note Springer Nature remains neutral with regard to jurisdictional claims in published maps and institutional affiliations.

\section{REFERENCES}

1. Vento, M. \& Saugstad, O. D. Targeting oxygen in term and preterm infants starting at birth. Clin. Perinatol. 46, 459-473 (2019).

2. Saugstad, O. D., Ramji, S., Soll, R. F. \& Vento, M. Resuscitation of newborn infants with 21 or $100 \%$ oxygen: an updated systematic review and meta-analysis. Neonatology 94, 176-182 (2008).

3. Vento, M. et al. Preterm resuscitation with low oxygen causes less oxidative stress, inflammation, and chronic lung disease. Pediatrics 124, e439-e449 (2009).

4. Kapadia, V. S. et al. Resuscitation of preterm neonates with limited versus high oxygen strategy. Pediatrics 132, e1488-e1496 (2013). 
Nitric oxide and preterm resuscitation: some words of caution M Vento and Ángel Sánchez-Illana

5. Wyckoff, M. H. et al. Part 13: neonatal resusciation 2015 American Heart Association guidelines update for cardiopulmonary resuscitation and emergency cardiovascular care. Circulation 132(Suppl. 2), S543-S560 (2015).

6. Oei, J. L. et al. Outcomes of oxygen saturation targeting during delivery room stabilization of preterm infants. Arch. Dis. Child Fetal Neonatal 103, F446-F454 (2018).

7. Sekar, K. et al. Inhaled nitric oxide as an adjunct to neonatal resuscitation in premature infants: a pilot, double blind, randomized controlled trial. Pediatr. Res. (2019). https://doi.org/10.1038/s41390-019-0643-x

8. Radi, R. Oxygen radicals, nitric oxide, and peroxynitrite: redox pathways in molecular medicine. Proc. Natl. Acad. Sci. USA 115, 5839-5848 (2018).

9. Sokol, G. M., Konduri, G. G. \& Van Meurs, K. P. Inhaled nitric oxide therapy for pulmonary disorders of the term and preterm infant. Semin. Perinatol. 40, 356-369 (2016).
10. Ballard, P. L. et al. Plasma biomarkers of oxidative stress: relationship to lung disease and inhaled nitric oxide therapy in premature infants. Pediatrics 121, 555-561 (2008).

11. Gasparovic, A. C., Zarkovic, N. \& Bottari, S. P. Biomarkers of nitro-oxidation and oxidative stress. Curr. Opin. Toxicol. 7, 73-80 (2018).

12. Thomas, C. A. \& Valentine, K. Utility of routine methemoglobin laboratory assays in critically ill pediatric subjects receiving inhaled nitric oxide. J. Crit. Care 48, 63-65 (2018).

13. Barrington, K. J., Finer, N. \& Pennaforte, T. Inhaled nitric oxide for respiratory failure in preterm infants. Cochrane Database Syst. Rev. 1, CD000509 (2017).

14. Dixon, F. et al. Treatment with nitric oxide in the neonatal intensive care unit is associated with increased risk of childhood cancer. Acta Paediatr. 107, 2092-2098. (2018). 